\title{
PARITY-VIOLATING NUCLEON-NUCLEON INTERACTIONS: WHAT CAN WE LEARN FROM NUCLEAR ANAPOLE MOMENTS?
}

\author{
B. DESPLANQUES* \\ LPSC, Université Joseph Fourier Grenoble 1, CNRS/IN2P3, INPG, \\ F-38026 Grenoble Cedex, France \\ *E-mail: desplanq@lpsc.in2p3.fr
}

\begin{abstract}
Knowledge about parity-violating effects both from theory and experiment is reviewed. Further information that could be obtained from measurements of nuclear anapole moments is discussed.
\end{abstract}

Keywords: Nucleon-nucleon forces, parity violation, anapole moments.

\section{Introduction}

In the present contribution, we are concerned with hadronic weak interactions and, more specifically, with the strangeness-conserving component. This one, contrary to the strangeness-violating one, is masked by strong (and electromagnetic) interactions. It can be disentangled through its parity-violating (pv) component, which leads to effects of order $10^{-7}$ for low-energy $N N$ interactions of interest here. Its experimental study is therefore expected to be difficult. It is however necessary to complement the knowledge from strangeness-violating processes. Until now, only the sector of nuclear interactions has been the object of experimental studies.

Similarly to the $N N$ strong interaction, the pv interaction is supposed to occur at the two-nucleon level in first place. Various approaches have been considered with different emphasis depending on time: more ambitious ones, based on meson exchange (see Refs. ${ }^{1,2}$ for instance), alternating with more phenomenological ones, devoted to the consistency of effects observed at low energy. ${ }^{3-6}$ In any case, the description of pv $N N$ interactions involves a minimal set of 5 pieces of information corresponding to the pv elementary $S-P$ transition amplitudes.

Looking at nuclear pv effects observed up to now, it is found that, for 
a large part, they individually agree with expectations within a factor $2 .^{7,8}$ This is not however sufficient to get a consistent description and, thus, a reliable determination of pv $N N$ forces. Further studies are required to provide the missing information. With this respect, we intent to review here some benchmark results, make comments partly in relation with recent works and show in what measurements of nuclear anapole moments could be useful. We largely refer to Ref. ${ }^{8}$ for omitted details.

The present paper is organized as follows. In Sec. 2, we review the description of the pv $N N$ forces in terms of meson exchanges. This includes a discussion of the numerous uncertainties pertinent to this approach. Section 3 is devoted to the phenomenological approaches considered in the past and recently. A possible hint for the failure of the single-meson exchange picture is described in Sec. 4. Presently known information is reviewed in Sec. 5. The conclusion describes in what the knowledge of nuclear anapole moments could be useful for getting a better determination of pv $N N$ forces.

\section{Meson-exchange description of $\mathrm{pv} N N$ forces}

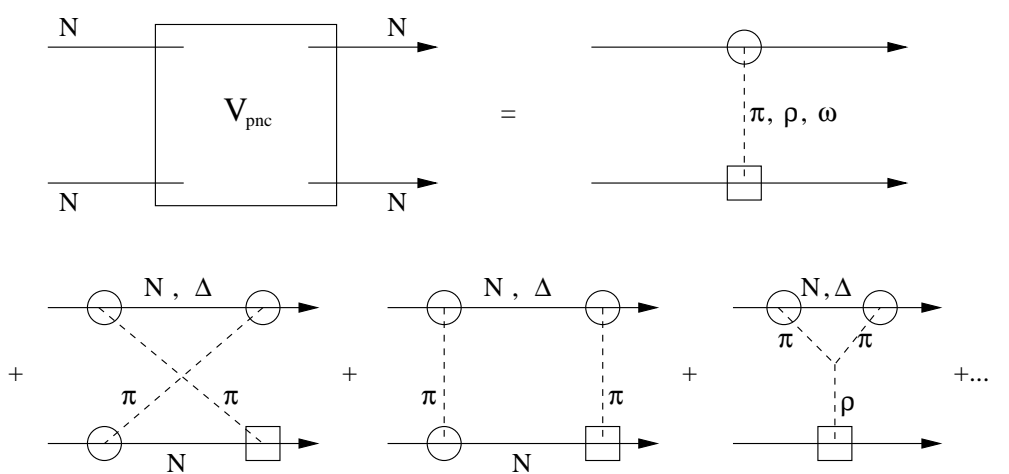

Fig. 1. Graphical representation of single-meson and some two-meson exchanges

Diagrams representing contributions to the pv $N N$ forces considered up to now are shown in Fig. 1. They involve the exchange of a single meson $(\pi, \rho, \omega)$ where the pv vertex (squared box) is described by a constant to be determined while the circle represents the strong-interaction vertex, in principle known. As the weak interaction does not conserve isospin, many couplings are possible in some cases. They are usually denoted as: $h_{\pi}^{1}: \quad \Delta T=1$ (long range, a priori favored), 
$h_{\rho}^{1^{\prime}}: \Delta T=1$ (short range),

$h_{\rho}^{0,1,2}: \Delta T=0,1,2$ (short range),

$h_{\omega}^{0,1}: \Delta T=0,1$ (short range).

The $\pi^{0}$ contribution is absent (Barton's theorem ${ }^{9}$ ) as well as that one due to other spin-0 mesons such as $\eta^{0}, \sigma^{0}$ (or two pions in a S state ${ }^{10}$ ).

Two-pion exchange contributions shown in Fig. 1 were considered in the 70 's, within a covariant approach. ${ }^{10-13}$ Some were recently considered at the dominant order within an effective field-theory framework. The dots in Fig. 1 stand for contributions due to heavier-meson, multi-meson or excitedbaryon exchanges. These contributions, which are expected to have a quite short range, were discarded in the 70's on the basis of a large repulsion in the $N N$ strong interaction models at these distances. This feature appears to be now a consequence of the local character of the models then used. ${ }^{14}$ The effect is much less pronounced with non-local models such as CDBonn ${ }^{15}$ or some Nijmegen ones. ${ }^{16}$ As a consequence, the support to neglect the above short-range pv contributions is now much weaker. On the other hand, these extra contributions could involve new parameters, making more complicated the description of pv $N N$ forces. One can only hope they are not too large.

From examining $N N$ strong interaction models, there are hints that the approximation of a single-meson exchange should be considered with caution. It has thus been shown that the combined effect of $\pi$ and $\rho$ exchange could provide some sizable repulsion. ${ }^{17}$ In its absence, the $\omega N N$ coupling has to be increased from an expected value of $g_{\omega N N}^{2} / 4 \pi \simeq 9$ to a value of about 20 . On the other hand, a model like Av18, ${ }^{18}$ which, apart from onepion exchange, is entirely phenomenological, fits experiments with a $\chi^{2}$ of 1 per data. This shows that the relevance of a single-meson exchange is far to be established. Thus, apart from $h_{\pi}^{1}$, pv couplings entering a single-meson exchange model based on $\pi, \rho, \omega$ could be quite effective.

Estimates for pv meson-nucleon couplings have been made by many authors. Most of them can be shown to be part of the DDH ones. ${ }^{1}$ We therefore rely on this work for a discussion. Some detail is given in Table 1 of Ref., ${ }^{8}$ the most important couplings being $h_{\pi}^{1}, h_{\rho}^{0}, h_{\omega}^{0}$. They are obtained from an effective $q q$ interaction, which depends on the factor $K \simeq 1+$ $\frac{25}{48 \pi^{2}} g^{2}(\mu) \log \left(\frac{M_{W}^{2}}{\mu^{2}}\right)$, which accounts for QCD strong-interaction effects. Part of the contributions is based on the $S U(6)_{W}$ symmetry and knowledge from non-leptonic hyperon decays. Another part is based on a factorization approximation. The original range assumed $1 \leq K \leq 6$, possible $S U(6)_{W}$ symmetry breaking and some weight for the neutral-current contribution. 
The best-guess values were obtained by weighting the various ingredients, in particular with respect to $S U(6)_{W}$ symmetry breaking and $q \bar{q}$-pair role. The update, which takes into account the fact that some ingredients are better known now, mainly affects the coupling $h_{\pi}^{1}$ (see Table 4 of Ref. ${ }^{8}$ ). Results from a quite different approach based on a soliton model $(\mathrm{KM})^{2}$ can also be found in Table 1 of Ref. ${ }^{8}$ Instead, results for $h_{\pi}^{1}$ obtained from QCD sum rules ${ }^{19-21}$ are not shown.

Examination of various results suggests many remarks. The comparison of DDH and KM results evidences interesting similarities despite differences in the approaches. In both cases, the contribution to $h_{\pi}^{1}$ due to non-strange quarks is suppressed, most of the estimate involving strange quarks. The estimates for $h_{\rho}^{0}$ and $h_{\omega}^{0}$ differ but rough agreement could be obtained by weighting differently the various contributions in DDH (notice that KM provides couplings at $q^{2}=0$ while DDH give them at the meson pole). On the basis of DDH estimates, the contribution of $h_{\omega}^{0}$ has often been neglected but KM results show it should not be. On the other hand, the upper limit for this coupling in $\mathrm{DDH}$ has not been reproduced. Its range should be mostly negative. As for the $h_{\pi}^{1}$ estimates from QCD sum rules, ${ }^{19-21}$ we notice that, as far as we can see, they do not correspond to any of the expected results shown in Table 4 of Ref. ${ }^{8}$ ). They are obtained from non-strange quarks only and involve a coherent sum of two contributions while cancellations are observed in DDH and KM. It is not clear at the present time whether they represent a new contribution or whether their relation to other results has not been found yet.

It has been mentioned that predictions for couplings should be corrected for various effects ${ }^{22}$ (rescattering, vertex corrections). These ones could affect the factorization part of DDH estimates but should not change the other parts which, being based on experimental non-leptonic hyperon decays, already account for them. The problem in this case is whether $S U(6)_{W}$ symmetry breaking effects are correctly accounted for.

\section{Phenomenological approaches to pv $N N$ forces}

For a part, phenomenological approaches have been motivated by the failure of single-meson-exchange approaches to provide a consistent description of various data. Less ambitious than the potential ones, they were originally limited to the five $S-P$ transition amplitudes. ${ }^{3,4}$ The important point is that their energy dependence in the few $\mathrm{MeV}$ range is completely deter-

mined by well-known strong-interaction properties. Thus, five parameters are required for their description. ${ }^{3,4}$ The approach was extended to higher 
energies, motivated by the fact that most effects known at that time were involving complex nuclei. ${ }^{5}$ In this aim, a 6th parameter, chosen as the $h_{\pi}^{1}$ coupling, was added. Due to its long range, the $\pi$-exchange force induced by this coupling indeed provides an extra sizable energy dependence for the ${ }^{3} S_{1}-{ }^{3} P_{1}$ transition amplitude beyond a few $\mathrm{MeV}$. Moreover, it contributes significant ${ }^{3} P_{1,2}-{ }^{3} D_{1,2}$ transitions in complex nuclei, with a sign opposite to the ${ }^{3} S_{1}-{ }^{3} P_{1}$ one. The configuration-space expression of the interaction can be found in Refs. ${ }^{5,8}$

The approach was considered again recently but within the framework of an effective-field theory. ${ }^{6}$ This one involves a chiral expansion in terms of a quantity $\Lambda_{\chi}^{-1}$. The number of parameters to describe the pv $N N$ interaction is the same as above. However, the part of two-pion exchange induced by the $h_{\pi}^{1}$ coupling at the first possible order, $\Lambda_{\chi}^{-3}$, is separated out with the motivation it is a medium-range interaction. At the same order, a specific contribution to the electromagnetic interaction appears, hence a 7th parameter in the approach. The momentum-space expression of the twopion exchange contribution can be found in Refs. ${ }^{6,23}$ It only contains two terms that correspond to the local ones of a more general expression considered in the 70's. ${ }^{10-13}$ Apart from some mistakes in the original work, the comparison with earlier works shows that: ${ }^{23}$ i) the leading-order approximation overestimates the results at finite distances, ii) the non-local terms that appear at the next order are not negligible, iii) in comparison to a $\rho$ exchange, the two-pion exchange has a longer range as expected from the two-pion tail but also a shorter range. In this case, the result is due to the fact that the two pions are exchanged in a $\mathrm{P}$ state. The exchange in a $\mathrm{S}$ state, which dominates at intermediate distances in the strong-interaction case, does not contribute here. ${ }^{10}$

For the purpose of analyzing experimental data, we introduce dimensionless quantities which are more closely related to the isospin properties of the system under consideration. ${ }^{5}$ They are $X_{p p}$ and $X_{n n}$, which involve the $p p$ and $n n$ forces, and $X_{p n}^{+}\left(+5.5 h_{\pi}^{1}\right), X_{p n}^{-}\left(-5.5 h_{\pi}^{1}\right), X_{p n}^{0}$, which involve the $p n$ force. The strengths $X_{p n}^{+}$and $X_{p n}^{-}$are appropriate for the description of the $p n$ force in odd-proton- and odd-neuton-nucleus forces while $X_{p n}^{0}$ does not contribute to them. The $\pi$-exchange contribution, is put between parentheses for the case it would be considered explicitly. We also introduce the combinations: $X_{N}^{p}=X_{p p}+X_{p n}^{+}\left(+5.5 h_{\pi}^{1}\right), X_{N}^{n}=X_{p p}+X_{p n}^{-}\left(-5.5 h_{\pi}^{1}\right)$, which determine the strengths of the proton- and neutron-nucleus forces (for a nucleus with spin and isospin equal to 0 ).

The $X$ parameters are closely related to the Danilov ones. ${ }^{5}$ We stress 
that they account for a lot of short-range unknown physics ( $N N$ shortrange correlations, heavy- and multi-meson exchange, relativity, $\cdots$ ). If necessary, they can be calculated in terms of the $h$ couplings entering a meson-exchange description of the pv $N N$ interaction, allowing one to check the underlying model. ${ }^{8,24}$

\section{Possible failure of the single-meson exchange model}

It has been proposed that the study of pv effects in $p p$ scattering at different energies could allow one to disentangle contributions due to $\rho$ and $\omega$ exchanges. There are measurements at $13.6 \mathrm{MeV}^{25}$ and $45 \mathrm{MeV},{ }^{26}$ which mainly involve a ${ }^{1} S_{0}-{ }^{3} P_{0}$ transition, and at $221 \mathrm{MeV},{ }^{27}$ which is sensitive to the ${ }^{3} P_{2}-{ }^{1} D_{2}$ transition. The analysis has been performed by Carlson et al., with the results: ${ }^{28} 10^{7} h_{\rho}^{p p}=-22.3,10^{7} h_{\omega}^{p p}=+5.2$.

The $\rho$ coupling agrees with DDH expectations while the $\omega$ one is at the extreme limit of the proposed range but disagrees if one notices that the range is now restricted to negative values. In short, it is found that the contribution to the ${ }^{3} P_{2}-{ }^{1} D_{2}$ transition is too small, roughly by a factor 2 . It has been thought that a longer-range force could enhance the contribution to the ${ }^{3} P_{2}-{ }^{1} D_{2}$ transition relatively to the ${ }^{1} S_{0}-{ }^{3} P_{0}$ one. ${ }^{29}$ While this is verified for the undistorted Born amplitude, it is not for the distorted one. Due to the effect of a strong short-range repulsion for the ${ }^{1} S_{0}-{ }^{3} P_{0}$ transition amplitude, the effect is the other way round, making the problem more severe. There are different issues: i) the measurement at $221 \mathrm{MeV}$ is too large (by a factor of about 2), ii) estimates of pv coupling constants miss important contributions, raising some doubt about present ones, iii) besides the single-meson exchange contribution, there are important extra contributions (multi-meson exchange, ...). For this last issue, there would be no other choice than to adopt an effective approach, in which case, the ${ }^{3} P_{2}-{ }^{1} D_{2}$ and ${ }^{1} S_{0}-{ }^{3} P_{0}$ transition amplitudes are described by independent parameters.

\section{Presently known information}

The first information we want to consider concerns the strength of the pv $p p$ force, $X_{p p}$. It can be obtained from measurements of the pv asymmetries $A_{L}$ in $p p$ scattering at 13.6 and $45 \mathrm{MeV}\left(A_{L}=-0.10\right.$ and $-0.17 X_{p p}$ respectively $\left.{ }^{30}\right)$. The fitted value: ${ }^{8}$

$$
X_{p p} \approx 0.9 \times 10^{-6}
$$


provides a very good description of the measurements, ${ }^{25,26}$ leaving little space for a ${ }^{3} P_{2}-{ }^{1} D_{2}$ transition, which is expected to be small in any case. It is stressed that the coefficients 0.10 and 0.17 are determined by well-known properties of the strong $N N$ interaction, while the unknown physics is incorporated in the quantity $X_{p p}$. The above value provides an unambiguous benchmark for the strength of pv $N N$ forces.

The second information is obtained from the strength of the protonnucleus force, $X_{N}^{p}$, mostly determined by pv effects observed in odd-proton systems such as $p-\alpha$ scattering or radiative transitions in complex nuclei $\left({ }^{19} \mathrm{~F},{ }^{41} \mathrm{~K},{ }^{175} \mathrm{Lu},{ }^{181} \mathrm{Ta}\right) .{ }^{8}$ While the dependence of most effects on $X_{N}^{p}$ results from the underlying nuclear model used in the estimate, ${ }^{31}$ this is not so in ${ }^{19} F$ where the estimate was based on a shell-model calculation. ${ }^{32}$ Examination of the detailed calculations in the last case nevertheless shows that the result has the structure of a single-proton transition in an average field determined by the strength $X_{N}^{p}$, evidencing the role of two effects that were accounted for in the heavier nuclei (pairing and deformation). Another calculation ${ }^{33}$ however suggests that correlations could affect differently isovector and isocalar contributions to $X_{N}^{p}$ (see also below). A good description of the observed effects assumes the fitted value: ${ }^{8}$

$$
X_{N}^{p} \approx 3.4 \times 10^{-6} \text {. }
$$

Examining the whole fit (within experimental errors), we are inclined to think it is too good however. At this point, we simply notice that the contribution of the $p p$ force to the proton-nucleus force is relatively small, pointing to a large contribution of the $p n$ force which could be due either to the isoscalar part of the pv force or to the isovector one.

The third available information concerns this isovector part of the force. It is obtained from the analysis of pv effects in the transition ${ }^{18} \mathrm{~F}\left(0^{-} \rightarrow\right.$ $\left.1^{+}\right)$. An extensive analysis of the effect in this process has been done. ${ }^{32}$ It essentially involves the difference of the pv proton- and neutron-nucleus forces and results in the following upper limit:

$$
\left|X_{N}^{p}-X_{N}^{n}\right| \leq 1.4 \times 10^{-6}
$$

from which we can derive a range relative to the neutron-nucleus force:

$$
2.0(2.6) \times 10^{-6} \leq X_{N}^{n} \leq 4.2(4.8) \times 10^{-6} .
$$

Assuming that the isovector contribution is dominated by the single-pion exchange force, one would get: $\left|h_{\pi}^{1}\right| \leq 1.3 \times 10^{-7}$. To some extent, the absence of effect in ${ }^{21} \mathrm{Ne}$ supports the above limit, which could even be smaller if the isoscalar contribution tends to be suppressed. ${ }^{34}$ To a lesser extent, results for ${ }^{75} T c^{35}$ lead to a similar conclusion. ${ }^{8}$ 
Concerning the other pieces of information, $X_{n n}$ and $X_{n p}^{0}$, one could rely on little details in the theoretical estimates to determine them from the analysis of pv effects in various processes. The comparison of these estimates, especially in complex nuclei, however shows that these details are somewhat uncertain, preventing one to get reliable information. The strength $X_{n n}$ would be best determined from measurements involving neutrons in light systems. The strength $X_{n p}^{0}$ does not play much role in complex nuclei. ${ }^{8}$ The most favorable process for its determination is the measurement of the photon circular polarization in the radiative capture $n+p \rightarrow d+\gamma$. Determining the 6 th parameter, $h_{\pi}^{1}$, introduced to get a better description of the pv $N N$ interaction at low energy, supposes to disentangle its long-range contribution from a short-range one. As the first contribution is expected to dominate the other one however, $h_{\pi}^{1}$ could be best determined from the measurement of the pv asymmetry in $\vec{n}+p \rightarrow d+\gamma$ where the effect is maximized (see Refs. ${ }^{8,23,36}$ and references therein). Determining the short-range part could be quite difficult in practice.

Though the information is incomplete, one can nevertheless have an interesting discussion relative to the strength of the $p p$ force, $X_{n n} \simeq 0.9 \times 10^{-6}$ and its contribution to the proton-nucleus one, $X_{N}^{p} \approx 3.4 \times 10^{-6}$. The relative sign is encouraging but the relative size supposes that a large contribution to $X_{N}^{p}$ comes from a $p n$ force. This is questionable in absence of a large pion-exchange contribution, as constrained from ${ }^{18} F\left(0^{-} \rightarrow 1^{+}\right)$. In usual potentials models, the isoscalar $p n$ contribution to $X_{N}^{p}$ is at best of the order of the $p p$ one. Thus, its strength could be larger than expected by a factor from 2 to 3 . This failure could indicate that the usual potential models miss some contribution, supporting for a part findings from $p p$ scattering. Interestingly, the analysis of pv effects in this process (see Sec. 4), with a positive $\omega N N$ coupling, tends to enhance the strength of the $p n$ force with respect to the $p p$ one. ${ }^{28,29}$ Another explanation supposes medium effects that could enhance the strength of the proton-nucleus pv force in heavy nuclei. Some mechanisms, in relation with an attraction in the isoscalar $0^{-}$ channel $^{8,33,37}$ (RPA correlations) or relativity, ${ }^{38}$ have been discussed in the literature but the size of the effect depends on poorly known ingredients.

\section{What from nuclear anapole moments? Conclusion}

In first approximation, nuclear anapole moments involve the strengths of the proton- and neutron-nucleus forces, $X_{N}^{p}$ and $X_{N}^{n}{ }^{39}$ Until now, there is no direct determination of the last one. Measurements of anapole moments in odd-neutron nuclei could therefore provide a valuable information on the 
strength $X_{N}^{n}$. This information could also be obtained from pv effects in $n-\alpha$ scattering (see Ref. ${ }^{40}$ and references therein), unless there are sizable medium effects. In such a case, the two measurements will complement each other and their comparison could allow one to determine the size of these corrections for which there is some hint in odd-proton nuclei.

The measurement of anapole moments in odd-proton nuclei has already been performed. In the most accurate case however $\left({ }^{133} \mathrm{Cs}^{41}\right)$, the strength of $X_{N}^{p}$ required to account for the measurement is roughly twice as much as that one given in Eq. (2), obtained from other odd-proton systems. ${ }^{42,43}$ A factor 2 is typical of theoretical nuclear uncertainties in estimates of pv effects in nuclei but, looking at different calculations, it sounds that the uncertainty is smaller in the case of ${ }^{133} \mathrm{Cs}$, hence some serious concern. Noticing that the strength of the proton-nucleus force could be larger than expected on the basis of its contribution due to the $p p$ force (see previous section), the result in ${ }^{133} \mathrm{Cs}$ could simply be explained by an enhancement effect that is already at work in other complex nuclei. If so, one could wonder why effects in heavy nuclei have not required such an effect, especially in ${ }^{41} \mathrm{~K}$ and ${ }^{175} \mathrm{Lu}$, where previous calculations were relatively stable. An explanation could be as follows. These calculations assumed that initial and final states are described by very simple configurations with some correlations (pairing, deformation) preserving the single-particle character of the pv transition. More detailed calculations, made later on in lighter nuclei on a similar basis, have shown that the weight of such a contribution is often decreased by the consideration of further correlations. ${ }^{32}$ In this case, some enhancement of the strength of the proton-nucleus force could also be required in ${ }^{41} \mathrm{~K}$ and ${ }^{175} \mathrm{Lu}$. Having shown that the anapole moment in ${ }^{133} \mathrm{Cs}$ does not necessarily contradict other pv effects in nuclei, provided that some medium effect is invoked, we believe that the measurement of anapole moments in other odd-proton complex nuclei could be helpful in clarifying the present understanding of pv effects in complex nuclei.

By looking at anapole moments of nuclei with different numbers of protons and neutrons, one could imagine to also determine the separate contributions to $X_{N}^{p}\left(X_{N}^{n}\right)$ due to $X_{p p}\left(X_{n n}\right)$ and $X_{p n}^{+}\left(X_{p n}^{-}\right)$. Involving smaller contributions, this program would however suppose that both measurements and theoretical estimates are very accurate. This could be an interesting program for a much further future, once strengths, $X_{N}^{p}$ and $X_{N}^{n}$, are unambiguously determined. 


\section{References}

1. B. Desplanques, J. F. Donoghue and B. R. Holstein, Ann. Phys. (N. Y.) 124, 449 (1980).

2. N. Kaiser and U.-G. Meissner, Nucl. Phys. A 499, 699 (1989).

3. G. S. Danilov, Sov. J. Nucl. Phys. 14, 443 (1972).

4. J. Missimer, Phys. Rev. C 14, 347 (1976).

5. B. Desplanques and J. Missimer, Nucl. Phys. A 300, 286 (1978).

6. S.-L. Zhu et al., Nucl. Phys. A 748, 435 (2005).

7. E. Adelberger and W. Haxton, Ann. Rev. Nucl. Part. Sci. 35, 501 (1985).

8. B. Desplanques, Phys. Rept. 297, 1 (1998).

9. G. Barton, Nuovo Cimento 19, 512 (1961).

10. M. Chemtob and B. Desplanques, Nucl. Phys. B 78, 139 (1974).

11. D. Pignon, Phys. Lett. 35B, 163 (1971).

12. B. Desplanques, Phys. Lett. 41B, 461 (1972).

13. H. Pirner and D. O. Riska, Phys. Lett. 44B, 151 (1973).

14. A. Amghar and B. Desplanques, Nucl. Phys. A 714, 502 (2003).

15. R. Machleidt, Phys. Rev. C 63, 024001 (2001).

16. V. G. J. Stoks et al., Phys. Rev. C 49, 2950 (1994).

17. J. W. Durso et al., Nucl. Phys. A 278, 445 (1977).

18. R. B. Wiringa, V. G. J. Stoks and R. Schiavilla, Phys. Rev. C 51, 38 (1995).

19. V. M. Khatsimovskii, Sov. J. Nucl. Phys. 42, 781 (1985).

20. E. M. Henley et al., Phys. Lett. B 367, 21 (1996).

21. W. H. P. Hwang, Z. für Physik C 75, 701 (1997).

22. S.-L. Zhu et al., Phys. Rev. D 63, 033006 (2001).

23. B. Desplanques et al., nucl-th/0803.2075.

24. C.-P. Liu, Phys. Rev. C 75, 065501 (2007).

25. P. D. Eversheim et al., Phys. Lett. B 256, 11 (1991).

26. S. Kistryn et al., Phys. Rev. Lett. 58, 1616 (1987).

27. A. R. Berdoz et al., Phys. Rev. Lett. 87, 272301 (2001).

28. J. Carlson et al., Phys. Rev. C 65, 035502 (2002).

29. C.-P. Liu, C. H. Hyun and B. Desplanques, Phys. Rev. C 73, 065501 (2006).

30. M. Simonius, Phys. Lett. 41B, 415 (1972), Nucl. Phys. A 220, 269 (1974).

31. B. Desplanques, Nucl. Phys. A 316, 244 (1979).

32. E. Adelberger et al., Phys. Rev. C 27, 2833 (1983).

33. M. Horoi and B. A. Brown, Phys. Rev. Lett. 74, 231 (1995).

34. B. Desplanques and O. Dumitrescu, Nucl. Phys. A 565, 818 (1993).

35. M. Hass et al., Phys. Lett. B 371, 25 (1996).

36. C. H. Hyun, S. Ando and B. Desplanques, Phys. Lett. B 651, 257 (2007).

37. V. V. Flambaum and O. K. Vorov, Phys. Rev. C 49, 1827 (1994).

38. C. J. Horowitz and O. Yilmaz, Phys. Rev. C 49, 3042 (1994).

39. V. V. Flambaum and I. B. Khriplovich, Sov. Phys. JETP 52, 835 (1980).

40. B. Desplanques, in Fundamental Physics with Pulsed Neutrons Beams (FPPNB-2000), eds. C. R. Gould et al. (World Scientific, 2000) pp. 87-96.

41. C. S. Wood et al., Science 275, 1759 (1997).

42. V. F. Dmitriev et al., Nucl. Phys. A 577, 691 (1994).

43. W. Haxton et al., Phys. Rev. C 65, 045502 (2002). 